Manuelle Medizin 2015 - 53:261-262

DOI 10.1007/s00337-015-0010-x

Online publiziert: 28. Juli 2015

(c) Springer-Verlag Berlin Heidelberg 2015

\author{
L. Beyer \\ Ärztehaus Mitte, Jena, Deutschland
}

\section{Die individuelle Erfahrung} Aufwertung durch Diskussion
Liebe Kolleginnen, liebe Kollegen,

eine Diskussion über die Bedeutung der Erfahrung für die Weiterempfehlung manueller Therapie zieht sich wie ein roter Faden durch die präsentierten Beiträge, obwohl das vorliegende Heft sich keiner spezifischen Thematik widmet.

Im Beitrag „Evidenz von Physiotherapie bei kraniomandibulärer Dysfunktion $(\mathrm{CMD})$ “ von Stelzenmüller et al. findet sich die Aussage: „Liegt die Ursache einer CMD in biomechanischen oder muskulären Funktionsstörungen, können die Beschwerden durch Physiound manuelle Therapie positiv beeinflusst werden. Durch eine begleitende physio-/manualtherapeutische Behandlung können bei Patienten mit CMD innerhalb der in den Heilmittelrichtlinien festgelegten zeitlichen Vorgaben von 15-20 min je Heilmittel gute bis sehr gute Ergebnisse erzielt werden. Physiotherapie ist besonders bei CMDSchmerzpatienten (Schmerzangabe $\geq 5$ auf einer Skala von 0 bis 10) hochwirksam." Dialektisch Sackett et al. zitierend, ergänzen die Autoren: „Externe klinische Evidenz kann individuelle klinische Erfahrung zwar ergänzen, aber niemals ersetzen. Es ist gerade diese individuelle Expertise, die entscheidet, ob die externe Evidenz überhaupt auf den einzelnen Patienten anwendbar ist und, wenn das zutrifft, wie sie in die Entscheidung integriert werden kann."

Es erscheint tatsächlich so, dass die manuelle Medizin ein hohes Potenzial für eine personalisierte Medizin hat, weil sie in der Lage ist, durch die manuelle Diagnostik einen individuellen spezifischen Befund am Bewegungssystem zu erheben und die Ursachen der Dysfunktion, die diesen Befund verursachen, aktuell patientenbezogen zu behandeln.
Daraus lässt sich aber nicht die Allgemeingültigkeit einer individuellen Erfahrung ableiten, auch wenn man vielleicht auf einen tausendfachen Behandlungserfolg im Laufe eines Praxislebens verweisen kann - es bleibt eine individuelle Erfahrung. Diese bedarf zur Multiplikation der genauen Analyse aller Begleitumstände und deren Diskussion. Sonst bleibt es beim „Glauben, der geholfen hat" oder eben auch nicht.

\section{》) Konsens nach Diskussion als erster Schritt zur Evidenz}

In Vorbereitung von Leitlinien zur Behandlung des spezifischen Rückenschmerzes wurden Vorschläge erarbeitet, die hier zur Diskussion gestellt werden.

Vorgestellt werden 2 Ansätze in der Form, wie sie in die Arbeitsgruppe zur Erstellung der Leitlinien eingebracht wurden:

1. die hypomobile segmentale Dys-

funktion der Lendenwirbelsäule

(Blockierung)

2. die myofasziale Dysfunktion

$\mathrm{Zu}$ diskutieren könnte sein, ob die beschriebenen Standpunkte für den chronischen Rückenschmerz nicht ein gemeinsames Substrat haben oder ob der Aspekt der pathogenetischen Zeitschiene in der Entwicklung eines chronischen Rückenschmerzes bzw. von Schmerzen im Bewegungssystem nicht subtiler zu betrachten ist. Möglicherweise liegt das therapeutische Optimum der manuellen Medizin in den Entstehungsphasen des Rückenschmerzes, vor der Chronifizierung.

Zwei Beiträge untersuchen die Relevanz etablierter Messverfahren. So stellen Prill u. Michel fest: „Bei der Bestimmung der sagittalen Kniegelenksbeweglichkeit werden bisher Gütekriterien weit weniger Beachtung geschenkt als individuellem Erfahrungswissen. Dieser Umstand führt bei Studien zur Beweglichkeitsförderung des Kniegelenks zwangsläufig zu nichtbelastbaren Interventionsergebnissen. "Laut Baumbach et al. sind validierte Messungen essenziell, um Diagnosen zu stellen oder Therapieerfolge zu quantifizieren.

Ein Beitrag von Heil et al. befasst sich noch einmal mit dem Zusammenhang zwischen Kiefergelenk und Körperhaltung, diesmal in umgekehrter Wirkungskette. Die Ergebnisse verdeutlichen die komplexen Zusammenhänge und Wirkungsmechanismen des menschlichen Bewegungssystems, die auch in der Praxis Berücksichtigung finden sollten. Die Autoren schlussfolgern, dass alle Bestimmungen der Kieferrelation und Bewegungsbahnen in einer sitzenden, aufrechten Körperposition vorgenommen werden sollten, um Fehlerquellen weitestgehend auszuschließen und eine hohe Genauigkeit der Messdaten zu erzielen.

Ein Bericht vom diesjährigen gemeinsamen Kongress des Zirkels für Manuelle Medizin und Entwicklungstherapie (ZiMMT) und der Ärztevereinigung für Manuelle Medizin (ÄMM) vermeldet neben einem intensiven fachlichen Austausch mit dem Auditorium auch Diskussionen zwischendurch in den Pausen und Abendveranstaltungen. Zur Diskussion lud das Thema „Osteopathie/ osteopathische Medizin/Manualmedizin bei Säuglingen und Kleinkindern - wie viel hilft wann wie viel?" Es wurde konstatiert, dass man sich als Praktiker kaum um die Sache an sich streite, sondern erst die Berufspolitik Brisanz schaffe. 


\section{Fachnachrichten}

Wie immer, aber diesmal besonders, sind wir auf Ihre Meinung in Leserzuschriften gespannt.

Beste kollegiale Grüße

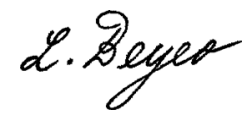

L. Beyer

\section{Korrespondenzadresse}

\section{Prof. Dr. L. Beyer}

Ärztehaus Mitte

Westbahnhofstr. 2, 07745 Jena, Deutschland lobeyer@t-online.de

Interessenkonflikt. L. Beyer gibt an, dass kein Interessenkonflikt besteht.

\section{Ihr Zugang zum Onlinearchiv}

Im Volltextarchiv auf springermedizin.de stehen Ihnen alle elektronisch verfügbaren Ausgaben Ihrer Zeitschrift zur Verfügung - unabhängig davon, seit wann Sie die Zeitschrift abonniert haben. Die Beiträge sind als PDF-Datei im Layout der gedruckten Ausgabe und als HTML-Version verfügbar. In der HTML-Version ist die Literatur verlinkt, sodass Sie direkt zu den zitierten Quellen gelangen können.

\section{Springer Medizin}

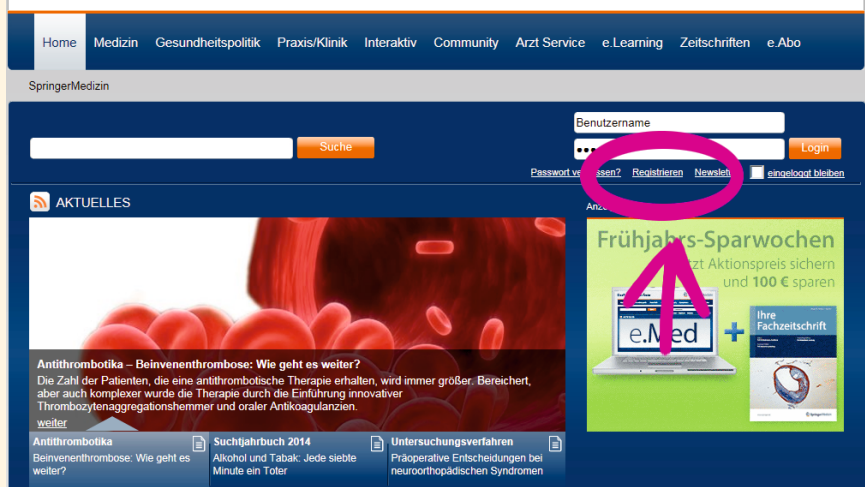

\section{Und so einfach geht es:}

\section{Einmalig registrieren:}

Registrieren Sie sich auf

www.springermedizin.de/manuelle-medizin über den Menüpunkt Registrieren (siehe Abbildung oben). Geben Sie dabei Ihre Abonummer an und ggf. die Adresse, an die Ihre Zeitschrift geliefert wird, sowie Ihre EFN-Nummer.

\section{Log in:}

Nach erfolgreicher Registrierung werden Ihnen Ihre Zugangsdaten per E-Mail zugeschickt.

\section{Ausgabenarchiv:}

Im Ausgabenarchiv unter

www.springermedizin.de/manuelle-medi- zin finden Sie alle elektronisch verfügbaren Ausgaben der Zeitschrift, sortiert nach Jahrgang und Ausgabennummer. Die einzelnen Beiträge werden im Inhaltsverzeichnis unter Angabe von Rubrik, Titel und Autoren übersichtlich angezeigt. Die Reihenfolge entspricht der im gedruckten Heft. Mit einem Klick gelangen Sie direkt zum gewünschten Beitrag.

Für alle Fragen zu Ihrem Onlinezugang steht Ihnen unser Kundenservice unter Kundenservice@springermedizin.de zur Verfügung. Telefonisch erreichen Sie die Hotline montags bis freitags von 9.00 bis $17.00 \mathrm{Uhr}$ kostenfrei unter 0800-77 80777 sowie gebührenpflichtig aus dem Ausland unter +4930884293600. 